\title{
MORDIDA ABERTA ANTERIOR - UMA REVISÃO DA LITERATURA
}

\author{
Tamyres Ruiz Abi ANTOUN ${ }^{1}$ \\ tamyres.abi@hotmail.com \\ Denis Clay Lopes dos SANTOS² \\ dr.denis@globomail.com \\ Everton FLAIBAN ${ }^{3}$ \\ evertonflaiban@hotmail.com \\ Daniel NEGRETE ${ }^{4}$ \\ daninegrete75@gmail.com \\ Renata BORTOLIN ${ }^{5}$ \\ renatabortolin@hotmail.com
}

Raquel Lopes dos SANTOS 6

raquellopesqls@hotmail.com

\section{RESUMO}

A mordida aberta anterior é vista como um dos maiores desafios dos ortodontistas; essa má oclusão ainda é muito discutida por sua origem multifatorial e por ser considerada de grande importância aos pacientes pelo fator estético. Essa causa ocorre por uma combinação variável, congênita ou adquirida. Classificar a etiologia da mordida aberta anterior e a idade do paciente é o primeiro passo, e de grande importância, para entender e solucionar essa má oclusão que acomete tanto o gênero masculino quanto feminino, principalmente na infância e durante a troca de dentição. A mordida aberta anterior (MAA) pode ser definida como a presença de um trespasse vertical negativo entre as bordas incisais dos dentes anteriores superiores e inferiores. Os profissionais da saúde precisam identificar precocemente a presença de hábitos bucais deletérios, pois a prevenção resulta em melhor qualidade de vida do paciente, favorecendo condições adequadas de alimentação, respiração e fala, melhorando harmonia e equilíbrio oral facial. Essa má oclusão, quando diagnosticada precocemente, aumenta as chances de sucesso da terapia ortodôntica.

DESCRITORES: MORDIDA ABERTA, MÁ OCLUSÃO, ORTODONTIA.

\footnotetext{
Aluna do curso de especialização em Ortodontia na Universidade Cruzeiro do Sul.

Professor Doutor coordenador do Curso de Odontologia da Universidade Cruzeiro do Sul.

Professor mestre dos cursos de pós-graduação da Universidade Cruzeiro do Sul.

Professor mestre dos cursos de pós-graduação da Universidade Cruzeiro do Sul.

Professora mestranda dos cursos de pós-graduação da Universidade Cruzeiro do Sul.

Professora mestranda dos cursos de pós-graduação da Universidade Cruzeiro do Sul. E-mail:
} 


\section{ANTERIOR OPEN BITE - A LITERATURE REVIEW}

\section{ABSTRACT}

The anterior open bite is considered one of the biggest challenges for orthodontists and this malocclusion is still much discussed because of its multifactor origin and because patients considered it of great importance by aesthetics factor. This cause occurs by a variable combination, congenital or acquired, classifying the etiology of anterior open bite and the age of the patient is the first step, one of great importance, to understand and solve this malocclusion that affects both male and female, especially during childhood and dentition exchange. The anterior open bite may be defined as the presence of a negative vertical overlap between the upper and lower anterior teeth's incisal edges. Health professionals need to identify early the presence of deleterious oral habits, the prevention results in a better life quality for the patient, because it favors adequate conditions of feeding, breathing and speech, improving harmony and oral facial balance. This malocclusion, when diagnosed early, increases the chances of successful orthodontic therapy.

DESCRIPTORS: OPEN BITE, MALOCCLUSION, ORTHODONTICS

\section{INTRODUÇÃO}

A mordida aberta anterior é assim classificada quando não há contato incisal em relação cêntrica, é definida como a presença de uma dimensão vertical negativa entre as bordas incisais dos dentes anteriores superiores e inferiores ${ }^{1}$. Os indivíduos com mordida aberta podem apresentar falta de contato entre os dentes, selamento labial deficiente, respiração bucal, fala atípica, atresia do arco maxilar, aumento do terço inferior da face, plano oclusal aumentado, corpo mandibular pequeno, maxila retruída e tendência a ser Classe II de Angle ${ }^{2}$. Essa má oclusão pode ser resultante de causas diversas, como: irrupção incompleta dos dentes anteriores, mau posicionamento da língua e presença de hábitos bucais deletérios ${ }^{3}$. É comum encontrar crianças que apresentam hábitos bucais, principalmente sucção, pressionamento lingual atípico e respiração bucal $^{4}$. A mordida aberta mais frequentemente observada na região anterior deve-se, principalmente, a hábitos, como sucção do polegar. Nesses casos, a mordida aberta geralmente apresenta-se de forma assimétrica, a posição dos dentes e a deformação dos processos alveolares exibem uma configuração que representa uma impressão negativa do polegar. A mordida aberta na região anterior também pode ser causada pelo posicionamento contínuo da parte anterior da língua entre as bordas incisais dos incisivos inferiores e da superfície lingual dos incisivos superiores. As mordidas abertas anteriores causadas exclusivamente por uma protrusão habitual da língua são geralmente simétricas ${ }^{5}$. 0 tratamento precoce proporciona melhores condições funcionais e estética, auxilia na prevenção de desarmonias ósseas severas e pode evitar intervenções cirúrgicas de maior complexidade ${ }^{6}$. As más oclusões verticais desenvolvemse como resultado de fatores etiológicos diversos, porém o padrão de crescimento desfavorável com predomínio vertical também pode levar à instalação desse quadro, ou mesmo favorecê-la ${ }^{3}$.

\section{METODOLOGIA}

Para a realização deste trabalho foi realizado um levantamento bibliográfico nas bases de dados Pubmed, SciELO e Bireme (BVS). Por ser uma revisão bibliográfica, os dados presentes foram coletados somente de documentos já existentes, não sendo necessário aplicar nenhuma pesquisa para levantamento de resultados. Os termos utilizados foram: "Mordida Aberta" e "Anterior Open Bite" e os critérios de inclusão foram: estudos epidemiológicos, estudos clínicos em humanos e relatos de caso clínico. 
ISSN 1983-5183

\section{REVISÃO DE LITERATURA}

Durante o desenvolvimento da oclusão, um conjunto de forças da mesma intensidade age sobre os dentes e componentes alveolares em direção vestíbulo-lingual. Essas forças pressionam o tecido ósseo que, embora considerado o segundo tecido mais duro do corpo humano, representa um tecido plástico, moldando a pressão exercida sobre ele ${ }^{7}$. O termo "mordida aberta" foi utilizado pela primeira vez por Caravelli, em 1842, como uma classificação distinta de má oclusão ${ }^{8}$. Alguns estudos foram realizados a fim de encontrar algumas classificações para essa má oclusão, assim como Worms et al. ${ }^{9}$ (1971), que classificaram a mordida aberta anterior em simples (compreendida de canino a canino, não incluindo os pré-molares e com $1 \mathrm{~mm}$ ou mais, em relação cêntrica), composta (de pré-molares a pré-molares, não incluindo os molares) e infantil, quando atinge os molares ${ }^{9}$. Kim classificou a mordida aberta em esquelética, que resulta em um padrão esquelético desfavorável, ocorrendo devido a alterações na maxila e/ou mandíbula e mordida aberta adquirida que apresenta um bom padrão facial, porém devido à interposição de língua e de hábitos de sucção ocorre a mordida aberta10. Dawson et al..11 (1974) classificam essa maloclusão, de acordo com a altura envolvida, em: (1) mínima, quando a abertura chega até 1mm; (2) moderada, de 1 a 5mm, e (3) severa, quando superior a $5 \mathrm{~mm}^{11}$.

Apesar de algumas classificações incluírem a divisão entre a mordida aberta dentária e esquelética, Nahoum destacou que essas más oclusões, quando de origem dentária, respondem bem à terapia miofuncional, e as de origem esquelética podem requerer método para controle vertical, como intrusão de molares, bite-blocks, mentoneiras e outros dispositivos ${ }^{12}$. Almeida et al. ${ }^{13}$ (1990) propuseram uma classificação da mordida aberta anterior de acordo com as estruturas afetadas, sendo elas dentárias, dentoalveolares e/ou esqueléticas. As de natureza dentária são resultantes da interrupção do desenvolvimento vertical normal dos dentes anteriores, sem o comprometimento do processo alveolar. Quando o processo alveolar é atingido, significa que a má oclusão evoluiu para dentoalveolar e, neste caso, há um comprometimento do desenvolvimento ósseo, pois o fator causal está bloqueando o seu curso normal de desenvolvimento; se uma mordida aberta dentária não for corrigida adequadamente, poderá evoluir e adquirir características esqueléticas ${ }^{13}$. Moyers e Riolu ${ }^{14}$ (1991) dividiram a mordida aberta em simples, quando a análise cefalométrica vertical não revela medidas anormais e o único problema é a falta de alguns dentes em encontrar a linha de oclusão e complexas, quando a análise cefalométrica vertical revela desarmonia nos componentes esqueléticos da altura facial (AFAl) ${ }^{14}$. A etiologia da mordida aberta anterior é multifatorial, pode estar associada a hábitos bucais deletérios e padrão de crescimento vertical ${ }^{15}$. Dentro de suas classificações, as do tipo dentária e dentoalveolar predominam por causas ambientais, como hábitos de sucção não nutritiva, respirador bucal e deglutição atípica com interposição de língua e alguns fatores de ação local como anquilose dentárias e distúrbios de irrupção; já no tipo esquelético predominam fatores genéticos, como padrão de crescimento ${ }^{16}$. Dentre esses fatores etiológicos, alguns merecem destaque pelo seu maior impacto em desenvolver a mordida aberta:

Hábitos Bucais de sucção: A prática de hábitos de sucção digital e de chupeta é considerada normal, porém o prolongamento dela pode resultar em uma má oclusão. Intensidade, duração, frequência, tipo de objeto e/ou órgão utilizado e a idade do início do hábito são os fatores que devem ser considerados para avaliar o impacto das alterações ${ }^{17}$. Existem várias hipóteses para explicar a etiologia do hábito de sucção não nutritiva, a clássica teoria psicanalítica de Freud, que considera a sucção digital como uma estimulação de prazer nas zonas erógenas da boca e dos lábios. A observação de recém-nascidos realizando a sucção não nutritiva de objetos sustenta a hipótese de que esse tipo de sucção não está ligado a fome e sim ao prazer, 
ANTOUN TRA, SANTOS DCL, FLAIBAN E, NEGRETE D, BORTOLIN R, SANTOS RL. Mordida Aberta Anterior - uma revisão da literatura. Rev. Odontol. Univ. Cid. São Paulo 2018 abr/jun 30(2) 190-199

ISSN 1983-5183

não constitui um hábito, mas uma necessidade instintiva ${ }^{18}$. 0 hábito de sucção dos dedos ou da chupeta até os três anos de idade consiste em um mecanismo de suprimento emocional e supera os prejuízos funcionais que acarreta e não deve sofrer interferências ${ }^{19}$. Entretanto, a persistência do hábito durante a fase inicial da dentadura mista deve ser considerada como deletéria, uma vez que os incisivos estão irrompendo e o hábito pode prejudicar o desenvolvimento normal da oclusão e do crescimento facial ${ }^{3}$. Quando os hábitos de sucção digital e chupeta desenvolvem a MAA em caráter dentoalveolar, a abertura da mordida é restrita à região de sucção. $O$ uso de chupeta apresenta-se na forma circular, já a sucção de polegar causa a inclinação dos incisivos superiores para vestibular e inferiores para lingual. Tanto a chupeta como o dedo, durante a sucção, interpõem-se entre os incisivos superiores e inferiores restringindo a erupção desses dentes, enquanto os dentes posteriores continuam a desenvolver-se no sentido vertical'. A instalação de uma má oclusão não depende apenas da existência do hábito, mas também do padrão de crescimento facial que a criança possui, bem como da duração, intensidade e frequência com que o hábito é realizado ${ }^{20}$. Nesses casos, a autocorreção da MAA pode ser obtida após a remoção do hábito de sucção, contanto que outras disfunções secundárias não tenham se instalado ${ }^{21}$. Essas disfunções secundárias podem se desenvolver devido à protrusão dos incisivos superiores gerada pelo hábito de sucção, dificultando o selamento necessário para a deglutição e fazendo com que a língua se posicione de forma anormal, principalmente em repouso"1.

Interposição lingual e deglutição atípica: A interposição lingual entre os arcos dentários durante a fonação, deglutição e durante o repouso, constitui uma anormalidade funcional denominada pressionamento lingual atípico e encontra-se presente em $100 \%$ dos casos com mordida aberta anterior ${ }^{22}$. Segundo Cavalcanti, a deglutição integra a fase final da mastigação e o início da digestão dos alimentos, representa um conjunto de movimentos que de início são voluntários e posteriormente involuntários ${ }^{23}$. A interposição de língua pode ser classificada como primária, determinando, assim, um pior prognóstico, pois consiste na causa principal do desenvolvimento da má oclusão, ou secundária, quando a língua apenas adapta-se a uma alteração morfológica já existente, causada pela sucção de dedos ou chupeta ${ }^{5}$, ${ }^{24}$. A interposição lingual pode ocorrer também nos casos de hipertrofia das tonsilas palatinas. 0 contato da porção posterior da língua com as amígdalas hipertrofiadas provoca uma sensação dolorosa e a língua acaba sendo projetada para frente e para baixo, interpondo-se entre os incisivo ${ }^{25}$. As mordidas abertas anteriores causadas exclusivamente por uma protrusão habitual da língua são geralmente simétricas, em contraste àquelas causadas por um hábito nocivo. Uma mordida aberta na região anterior também pode ser causada pelo posicionamento contínuo da parte anterior da língua entre as bordas incisais dos incisivos inferiores e da superfície lingual dos incisivos superiores ${ }^{26}$. Durante a infância, a língua é proporcionalmente maior do que a cavidade bucal e, por isso, se protrui além dos rebordos alveolares. 0 crescimento dos ossos maxilares ao longo da infância é maior do que o da língua e, assim, o tamanho da cavidade bucal será adequado para o seu tamanho. Deve-se ressaltar que, se existe interposição de língua, seja ela postural ou durante a deglutição e fonação, e este hábito persistir após a correção da má oclusão, há uma grande chance de recidiva da mordida aberta anterior, sendo necessária a indicação do paciente ao tratamento fonoaudiológico, para que a terapia de reeducação muscular seja realizada ${ }^{24}$.

Respiração Bucal: A importância da função respiratória no desenvolvimento das estruturas orofaciais tem sido discutida e, de acordo com a teoria da "Matriz Funcional de Moss", a respiração nasal permite um adequado crescimento e desenvolvimento do complexo craniofacial interagindo com outras funções como mastigação e deglutição. Essa teoria baseia-se no princípio de que o crescimento facial está intimamente ligado à atividade funcional, representada por diferentes componentes da área da cabeça e pescoço $0^{27}$. 
ANTOUN TRA, SANTOS DCL, FLAIBAN E, NEGRETE D, BORTOLIN R, SANTOS RL. Mordida Aberta Anterior - uma revisão da literatura. Rev. Odontol. Univ. Cid. São Paulo 2018 abr/jun 30(2) 190-199

ISSN 1983-5183

Um estudo de Silva Filho et al. ${ }^{28}$, publicado em 1991, mostrou que a respiração predominantemente bucal acompanha cerca de $83 \%$ dos casos de mordida aberta anterior. A explicação é que a língua não pressiona o palato, permanece em uma posição mais abaixada, e a mandíbula fica posicionada mais pósteroinferiormente, permitindo a irrupção passiva dos dentes posteriores, gerando um aumento da altura facial ântero-inferior (AFAl) e da convexidade do perfi|28. A respiração bucal exige uma mudança postural para obter a abertura da via aérea oral, essas alterações favorecem um maior desenvolvimento ântero-inferior da face, assim como a atresia do arco dentário superior e a mordida aberta anterior. ${ }^{16}$ Outros fatores que desencadeiam a respiração bucal e a inter-relação entre a forma e a função são as obstruções das vias aéreas superiores (naso e bucofaringe) que podem ser causadas por hipertrofia da adenoide ou amígdalas, desvio de septo, rinites alérgicas e coriza crônica. Para a realização da respiração bucal, nos casos onde existe uma obstrução da nasofaringe, deverá ocorrer uma adaptação funcional com o abaixamento da mandíbula, posicionando a língua anteriormente e depositada no assoalho da cavidade bucal. Portanto, é a alteração postural da língua e mandíbula que provoca modificações aos níveis dentário e ósseo ${ }^{29}$. Como consequência da obstrução nasal, a face apresenta características peculiares, tais como a face torna-se longa e estreita, lábio superior hipotônico, curto e elevado, lábios separados e ressecados, nariz pequeno, afilado ou estreito e olheiras profundas ${ }^{30}$.

Padrão de Crescimento: A hereditariedade é um fator de extrema importância na etiologia da MAA, pois são os genes que controlam o padrão de crescimento. Quanto mais vertical for o vetor de crescimento, maior será a probabilidade de existir uma tendência a mordida aberta anterior ${ }^{31}$. Um paciente com tendência ao crescimento vertical apresenta rotação mandibular no sentido horário, além das características estruturais definidas por Björk ${ }^{32}$ (1969), tais como inclinação da cabeça do côndilo, curvatura do canal mandibular, rotação mandibular horária, ângulo goníaco aberto, ramo mandibular curto, divergência entre os planos palatino e mandibular, superirrupção dos dentes póstero-superiores, aumento na altura facial anterior inferior $(\mathrm{AFAl})^{32}$.

O desenvolvimento de uma mordida aberta anterior correlaciona o padrão de crescimento esquelético, e pacientes braquifaciais que possuem predominância de crescimento horizontal dificilmente desenvolverão este tipo de má oclusão. Nos mesofaciais, que possuem crescimento equilibrado, a mordida aberta até pode se desenvolver, mas com possibilidades de autocorreção, caso não apresentarem hábitos secundários. Porém os pacientes dolicofaciais apresentam um crescimento facial vertical, são pacientes predispostos a esse tipo de má oclusão e a sua gravidade pode ser aumentada pela ocorrência de hábitos de sucção, deglutição atípica e respiração bucal ${ }^{33}$.Em 1964, Subtelny e Sakuda ${ }^{34}$ publicaram um artigo sobre o diagnóstico e o tratamento da MAA. Partindo da premissa de que nos adolescentes os hábitos funcionais anormais diminuem ou desaparecem, esses autores queriam uma explicação para a existência do que eles chamaram de "mordidas abertas persistentes", isto é, aquelas que persistiam após a infância. Eles realizaram um estudo cefalométrico em 25 pacientes com "mordidas abertas persistentes" e os compararam com 30 pacientes com oclusão normal; todos os indivíduos tinham mais de 12 anos de idade. Nos casos de mordida aberta, foram verificadas basicamente as seguintes diferenças significativas: maior erupção dos molares superiores, assim como extrusão dos incisivos superiores, e planos mandibulares e ângulos goníacos excessivamente aumentados. Esse padrão facial passou a ser chamado de "mordida aberta esquelética", tendo como principal fator etiológico um padrão de crescimento desfavorável, com divergência das bases ósseas e falta de contato entre os incisivos ${ }^{34}$. Devido à etiologia multifatorial, existem diversos tipos de abordagem para tratamento da MAA. Vários protocolos foram introduzidos para o tratamento de pacientes com MAA, considerando-se a idade, fatores etiológicos e padrão de má oclusão ${ }^{35}$ e ainda não existe um 
ANTOUN TRA, SANTOS DCL, FLAIBAN E, NEGRETE D, BORTOLIN R, SANTOS RL. Mordida Aberta Anterior - uma revisão da literatura. Rev. Odontol. Univ. Cid. São Paulo 2018 abr/jun 30(2) 190-199

ISSN 1983-5183

consenso a respeito de qual é o melhor tratamento para essa má oclusão. Os diferentes tipos de tratamento podem incluir: (a) modificar comportamento para eliminar hábitos anormais; (b) movimentação ortodôntica através da extrusão de dentes anteriores ou intrusão de molares; e (c) tratamento cirúrgico das bases ósseas ${ }^{36}$.

Tratamentos Funcionais: A terapia miofuncional é utilizada para a correção dos hábitos e consiste em exercícios para reeducar a musculatura orofacial na deglutição, fonação e posição postural de descanso. Outra forma de corrigir hábitos funcionais é através de aparelhos que impeçam que a língua se apoie sobre os dentes; os mais conhecidos são as grades palatinas e os esporões. Esses aparelhos devem ser fixos para reeducarem a função até obter-se a automação do movimento ${ }^{21}$. As grades palatinas ou linguais são indicadas para corrigir a MAA, pois impendem que a língua se apoie sobre os dentes, precisam ser longas para evitar que a língua se posicione abaixo delas. Porém, são estruturas lisas e permitem que a língua fique apoiada sobre a grade ${ }^{8,17}$. Os esporões forçam uma mudança na postura de repouso da língua, que modifica a percepção sensorial do cérebro, obtendo-se, assim, uma nova resposta motora. Essa resposta pode ficar impressa definitivamente no cérebro, o que explica a possível mudança permanente da postura lingual produzida pelos esporões ${ }^{8}$.

Tratamentos Ortodônticos: Existem vários tipos de tratamento, por movimentação ortodôntica, para a correção da mordida aberta, com diferentes objetivos terapêuticos. A utilização de aparelhos extrabucais, mentoneiras verticais, bite-blocks e aparelhos funcionais têm como objetivo reduzir a extrusão de molares ${ }^{37}$. Mecânicas de elásticos intrabucais são utilizadas tanto para a extrusão de incisivos como para a intrusão de molares ${ }^{38}$. Todos esses aparelhos têm como objetivo inibir fatores mecânicos que mantêm a mordida aberta (interposição de língua e sucção de dedos). Todavia, há controvérsias envolvendo os efeitos dentoesqueléticos e em tecidos moles que esses tratamentos podem acarretar ${ }^{39}$. Além desses recursos de tratamentos interceptores, o tratamento ortodôntico corretivo por meio de aparelhos fixos pode corrigir essa malocusão utilizando dispositivos de ancoragem temporária, como mini-implante, sendo um eficiente procedimento biomecânico para corrigir até severas mordidas abertas ${ }^{40}$. Em pacientes adolescentes e adultos, o tratamento ortopédico apresenta grande limitação por causa da ausência do potencial de crescimento, sugerindo que o tratamento seja realizado principalmente pela compensação dentária ou, em casos graves, auxiliado pela cirurgia ortognática ${ }^{41}$.

Tratamentos Cirúrgicos: Esta deformidade dentofacial é conhecida por sua dificuldade de tratamento e pouca previsão da estabilidade no período pós-operatório em relação às demais deformidades ${ }^{42}$. 0 tratamento ortocirúrgico de escolha para a mordida aberta esquelética tem se modificado ao longo dos anos, sendo a estabilidade pós-cirúrgica um dos fatores mais decisivos na sua escolha. Os procedimentos cirúrgicos incluem osteotomias maxilares, mandibulares, uma combinação de ambas e a osteotomia alveolar $^{43}$. O tipo de cirurgia escolhida pode variar desde um avanço linear unimaxilar relativamente simples, até uma complexa cirurgia tridimensional bimaxilar, dependendo da deformidade esquelética ${ }^{44}$. Denison et al. ${ }^{45}$ (1989) avaliaram a estabilidade do tratamento cirúrgico na mordida aberta anterior em 66 pacientes adultos acompanhados por pelo menos um ano pós-cirurgia. Esses pacientes foram divididos de acordo com o trespasse vertical pré-cirúrgico: mordida aberta, mordida aberta com trespasse e trespasse normal. A mordida aberta recidivou $42,9 \%$ dos casos do grupo mordida aberta, enquanto que os grupos mordida aberta com trespasse e trespasse normal não apresentaram modificações pós-cirurgia. Foi observado que a instabilidade encontrada nos pacientes do grupo mordida aberta ocorreu devido a modificações dentoalveolares e não a modificações esqueléticas ${ }^{45}$. Acredita-se que as recidivas encontradas em pacientes tratados cirurgicamente sejam de origem dentoalveolar, geradas por disfunções bucais, já que esses 
ANTOUN TRA, SANTOS DCL, FLAIBAN E, NEGRETE D, BORTOLIN R, SANTOS RL. Mordida Aberta Anterior - uma revisão da literatura. Rev. Odontol. Univ. Cid. São Paulo 2018 abr/jun 30(2) 190-199

ISSN 1983-5183

pacientes são adultos e apresentam crescimento mínimo ou nulo ${ }^{26}$. Esses dados sugerem que a recidiva da mordida aberta anterior é causada, em geral, pela postura anterior de língua em repouso, um fator etiológico que não tem recebido a devida atenção nos tratamento cirúrgicos ${ }^{8}$.

\section{CONCLUSÕES}

O tratamento precoce da mordida aberta proporciona melhores condições funcionais e estéticas, podendo ser feito nas fases de dentição decídua, mista e permanente. Entretanto, o tratamento para esse tipo de anomalia deve ser precoce, para prevenir desarmonias ósseas severas e evitar intervenções cirúrgicas de maior complexidade; eliminando-se todos os seus fatores etiológicos, a estabilidade da correção aumenta significativamente, devido à manutenção do equilíbrio entre as estruturas do sistema estomagnático. Para o tratamento mais eficaz, é necessária atuação multidisciplinar entre o ortodontista, o otorrinolaringologista e o fonoaudiólogo, os quais, após avaliação, irão encaminhar o paciente para os especialistas das demais áreas (psicologia, pediatria e fisioterapia), caso seja pertinente.

\section{REFERÊNCIAS}

1. BURFORD D, Noar JH. The causes, diagnosis and treatment of anterior open bite. Dental update 2003 Jun;30(5):235-41.

2. MACIEL CTV, Leite ICG. Aspectos etiológicos da mordida aberta anterior e suas implicações nas funções orofaciais. Pró-Fono Rev Atual Cient 2005 17(3):293-302.

3. NGAN P, Fields HW. Open bite: a review of etiology and management. Pediatric dentistry 1997 Mar-Apr;19(2):91-8.

4. SERRA-NEGRA JMC, Pordeus IA, Rocha Jr JF. Estudo da associação entre aleitamento, hábitos bucais e maloclusões. Rev Odontol Univ São Paulo 1997 11(2):79-86.

5. PROFFIT WR. Ortodontia contemporânea. 5. ed. Rio de Janeiro: Guanabara Koogan 2013.

6. MONGUILHOTT LMJ, Frazzon JS, Cherem VB. Hábitos de sucçäo: como e quando tratar na ótica da ortodontia X fonoaudiologia. Rev dent press ortodon ortop maxilar 2003 jan.-fev. ;8(1):95-104.

7. NAKAO TH, Bertos FA, Oliveria DT, Bartoz AP, Bigliazzi R. Hábitos bucais como fatores de risco para a mordida aberta anterior: uma revisão de literatura. Rev Odontol Araçatuba 2016 maio-ago.;37(2):9-16.

8. ARTESE A, Drummond S, Nascimento JM, Artese F. Critérios para o diagnóstico e tratamento estável da mordida aberta anterior. Dental Press J Orthod 2011 jun.;16(3):136-61.

9. WORMS FW, Meskin LH, Isaacson RJ. Open-bite. American journal of orthodontics 1971 Jun;59(6):589-95.

10. KIM YH. Overbite depth indicator with particular reference to anterior open-bite. Am J Orthod. 1974 Jun;65(6):586-611.

11. DAWSON PE. Solving anterior open-bite problem. Saint Louis: Mosby; 1974. 
ANTOUN TRA, SANTOS DCL, FLAIBAN E, NEGRETE D, BORTOLIN R, SANTOS RL. Mordida Aberta Anterior - uma revisão da literatura. Rev. Odontol. Univ. Cid. São Paulo 2018 abr/jun 30(2) 190-199

ISSN 1983-5183

12. NAHOUM HI. Vertical proportions: a guide for prognosis and treatment in anterior open-bite. Am J Orthod. 1977 Aug;72(2):128-46.

13. ALMEIDA RR, Ursi WJ. Anterior open bite. Etiology and treatment. Oral health 1990 Jan;80(1):27-31.

14. MOYERS RE, Riolu ML. Tratamento precoce. In: Moyers, RE. Ortodontia. 4. ed. Rio de Janeiro: Guanabara Koogan; 1991. p. p. 292-369.

15. CARVALHO CM, Carvalho LFPC, Forte FDS, Aragao MS, Costa LJ. Prevalência de mordida aberta anterior em crianças de 3 a 5 anos em Cabedelo/PB e relação com hábitos bucais deletérios. Pesq Bras Odontoped Clin Integr João Pessoa 2009 9(2):205-10.

16. HENRIQUES JFC, Janson GRP, Almeida RR, Dainesi EA, Hayasaki SM. Mordida aberta anterior: a importância da abordagem multidisciplinar e consideraçöes sobre etiologia, diagnóstico e tratamento: apresentaçäo de um caso clínico. Rev dent press ortodon ortop maxilar 2000 maio-jun.;5(3):29-36.

17. TORRES F, Almeida RR, De Almeida MR, Almeida-Pedrin RR, Pedrin F, Henriques JF. Anterior open bite treated with a palatal crib and high-pull chin cup therapy. A prospective randomized study. Eur j orthod. 2006 Dec;28(6):610-7.

18. TANAKA O, Kreia TB, Bezerra JGB, Maruo H. A má-oclusão e o hábito de sucção de diferentes dedos. J Bras Ortodon Ortop Facial 2004 9(51):276-83.

19. GOIS EGO. A influência dos hábitos de sucção não nutritiva, do padrão respiratório e do tamanho da adenoide no desenvolvimento das más oclusões na dentição decídua: estudo tipo caso-controle em pré-escolares de Juiz de Fora - MG [Dissertação]. Belo Horizonte, MG: Universidade Federal de Minas Gerais; 2005.

20. GRABER TM. Ortodoncia: teoría y práctica. 3. ed. . In: Graber, TM. Etiología de la maloclusión: factores locales. México: InterAmericana; 1974. p. p.311-74.

21. FRANCO FCM, Araújo TM, Habib F. Pontas ativas: um recurso para o tratamento da mordida anterior Ortodon gaúch 2001 jan.-jun. ;5(1):6-12.

22. ALMEIDA RR, Almeida Pedrin RR, Almeida MR, Garib DG, Almeida PCMR, Pinzan A. Etiologia das más oclusöes: causas hereditárias e congênitas, adquiridas gerais, locais e proximais (hábitos bucais). Rev dent press ortodon ortop maxilar 2000 nov.-dez. ;5(6):107-29.

23. CAVALCANTI RVA. Más oclusões $X$ alteralções oromiofuncionais [Monografia]. Rio de Janeiro: : Cefac-Centro de Especializações em Fonoaudiologia Clínica- Motricidade Oral; 1999.

24. JUSTUS R. Correction of anterior open bite with spurs: long-term stability. World J Orthod 2001 2(3):219-31.

25. REIS MJ, Pinheiro CN, Malafaia M. Tratamento da mordida aberta anterior: relato de caso clínico. Rev Clín Ortodon Dental Press, Maringá 2007 ago.-set.;6(4):88-96.

26. MONTANARE M. Mordida aberta anterior [Monografia]. São Paulo: Faculdade de Odontologia, Universidade Estadual Paulista, Araçatuba; 2013. 
ANTOUN TRA, SANTOS DCL, FLAIBAN E, NEGRETE D, BORTOLIN R, SANTOS RL. Mordida Aberta Anterior - uma revisão da literatura. Rev. Odontol. Univ. Cid. São Paulo 2018 abr/jun 30(2) 190-199

ISSN 1983-5183

27. SILVA LK, Brasolotto AG, Berretin-Felix G. Função respiratória em indivíduos com deformidades dentofaciais. Rev CEFAC 2015 17(3):854-63.

28. DA Silva Filho OG, Gomes Gloncalves RJ, Maia FA. Sucking habits: clinical management in dentistry. J clin pediatr dent. 1991 Spring;15(3):137-56.

29. ALMEIDA RR, Almeida-Pedrin RR, Almeida MR, Ferreira FPC, Pinzan A, Insabralde CMB. Displasias verticais: mordida aberta anterior - tratamento e estabilidade. Rev dent press ortodon ortop maxilar 2003 jul.-ago. ;8(4):91-119.

30. CINTRA CFSC, Castro FFM, Cintra PPVC. As alteraçôes orofaciais apresentadas em pacientes respiradores bucais. Rev bras alergia imunopatol 2000 mar.-abr. ;23(2):78-83.

31. CASSIS MA. Tratamento da mordida aberta anterior com esporão colado e mentoneira: estudo comparativo dos efeitos dentoalveolares e esqueléticos [Dissertação]. São Paulo: Universidade de São Paulo; 2009.

32. BJORK A. Prediction of mandibular growth rotation. American journal of orthodontics 1969 Jun;55(6):585-99.

33. ALMEIDA RR, Santos SCBN, Santos ECA, Insabralde CMB, Almeida MR. Mordida aberta anterior: considerações e apresentação de um caso clínico. Rev Dental Press Ortodon Facial 1998 março-abr.;3(2):17-29.

34. SUBTELNY JD, Sakuda M. Open-bite: diagnosis and treatment. Am J Orthod. 1964 50(5):337-58.

35. FABRE AF, Mendonça MR, Cuoghi OA, Farias APF. Mordida aberta anterior: considerações-chave. Arch Health Invest 2014 3(5):48.

36. ZUROFF JP, Chen SH, Shapiro PA, Little RM, Joondeph DR, Huang GJ. Orthodontic treatment of anterior open-bite malocclusion: stability 10 years postretention. Am J Orthod Dentofac Orthop. 2010 Mar;137(3):302.e1-8; discussion -3.

37. BALLANTI F, Franchi L, Cozza P. Transverse dentoskeletal features of anterior open bite in the mixed dentition. Angle Orthod. 2009 Jul;79(4):615-20.

38. GREENLEE GM, Huang GJ, Chen SS, Chen J, Koepsell T, Hujoel P. Stability of treatment for anterior open-bite malocclusion: a meta-analysis. Am J Orthod Dentofac Orthop. 2011 Feb;139(2):154-69.

39. MILLER H. The early treatment of anterior open bite. Int j orthod. 1969 Mar;7(1):5-14.

40. ERVERDI N, Tosun T, Keles A. A new anchorage site for the treatment of anterior open bite: zygomatic anchorage case report. World J Orthod 2002 3(2):147-53.

41. LIMA GN, Cordeiro CM, Justo JS, Rodrigues LCB. Mordida aberta anterior e hábitos orais em crianças. Rev Soc Bras Fonoaudiol 2010 15(3):369-75.

42. MEDEIROS RB. A estabilidade do tratamento da mordida aberta em pacientes adultos [Dissertação]. Rio de Janeiro: Universidade Federal Fluminense; 2011.

43. REYNEKE JP, Ferretti C. Anterior open bite correction by Le Fort I or bilateral sagittal split osteotomy. Oral and maxillofacial surgery clinics of North America 2007 
ANTOUN TRA, SANTOS DCL, FLAIBAN E, NEGRETE D, BORTOLIN R, SANTOS RL. Mordida Aberta Anterior - uma revisão da literatura. Rev. Odontol. Univ. Cid. São Paulo 2018 abr/jun 30(2) 190-199

ISSN 1983-5183

Aug;19(3):321-38, v.

44. MCCANCE AM, Moss JP, James DR. Stability of surgical correction of patients with Skeletal III and Skeletal II anterior open bite, with increased maxillary mandibular planes angle. Eurj orthod. 1992 14(3):198-206.

45. DENISON TF, Kokich VG, Shapiro PA. Stability of maxillary surgery in openbite versus nonopenbite malocclusions. Angle Orthod. 1989 Spring;59(1):5-10.

RECEBIDO EM 03/01/2018

ACEITO EM 04/04/2018 